\title{
Observing single-atom diffusion at a molecule-metal interface
}

\author{
Johannes Mielke, ${ }^{1, *}$ Jesús Martínez-Blanco, ${ }^{1, \dagger}$ Maike V. Peters, ${ }^{2}$ Stefan Hecht, ${ }^{2}$ and Leonhard Grill ${ }^{1,3, \ddagger}$ \\ ${ }^{1}$ Department of Physical Chemistry, Fritz-Haber Institute of the Max-Planck Society, Faradayweg 4-6, 14195 Berlin, Germany \\ ${ }^{2}$ Chemistry Department, Humboldt-Universität zu Berlin, Brook-Taylor-Strasse 2, 12489 Berlin, Germany \\ ${ }^{3}$ Department of Physical Chemistry, University of Graz, Heinrichstrasse 28, 8010 Graz, Austria \\ (Received 24 February 2016; revised manuscript received 20 June 2016; published 12 July 2016)
}

\begin{abstract}
The dynamics at the interface between a close-packed porphyrin monolayer and $\mathrm{Au}(111)$ is investigated by time-dependent scanning tunneling microscopy, detecting the motion of single-interface adatoms in real space. Imaging sequences reveal predominant switching of the molecular appearance in adjacent molecules, pointing to a spatial correlation that is consistent with adatom diffusion from one molecule to the next. In some cases, the number of switching molecules is drastically increased, indicating collective switching events. In addition to the thermally induced motion of adatoms at the interface, also voltage pulses from the microscope tip can induce the process - revealing different yields in agreement with the model of adatom hopping.
\end{abstract}

DOI: 10.1103/PhysRevB.94.035416

The interface between organic molecules and metal substrates plays a key role for many properties that are important, for instance, in catalytically driven reactions [1,2] and for charge transport [3]. The study of such systems gives detailed insight into fundamental processes that take place at an organic-inorganic interface. For an in-depth understanding, it is advantageous to use a scanning tunneling microscope (STM) for imaging because it provides information about the local environment of each individual molecule, which is important since the molecular properties might differ locallydue to defects, step edges, or different areas of a surface reconstruction [4,5]. For instance, individual $\mathrm{C}_{60}$ molecules were found to change between dark and bright appearances in STM images, caused by the diffusion of surface [6] or bulk [7] vacancies, charge transfer between molecule and surface [8], or adsorption on small metal islands [9].

Adatoms are known to diffuse on close-packed metal surfaces if the thermal energy is sufficient for their detachment from step edges [10]. When molecules are adsorbed on such a surface, the adatoms can interact with and even be trapped by molecules [11-15], leading to characteristic metallic nanostructures that reflect the molecular shape [16-18]. In addition to these self-organized structures, manipulation of single adatoms has been used to bring them in contact with molecules [19,20]. So far, real space studies of the molecule-surface interaction have been done mainly under static conditions $[21,22]$ or focus on the lateral diffusion of molecules on surfaces [23-25]. Here, we report the dynamic behavior at the molecule-metal interface by observing the motion of individual gold adatoms underneath a molecular monolayer on $\mathrm{Au}(111)$ in time.

As a molecular system we have chosen tetrabromophenylporphyrin [Br ${ }_{4}$ TPP; Fig. 1(a)], deposited on $\mathrm{Au}(111)$. Experiments were done with a variable temperature STM at room temperature (see the Supplemental Material

\footnotetext{
*Present address: BAM Bundesanstalt für Materialforschung und -prüfung, Berlin, Germany.

${ }^{\dagger}$ Present address: Paul-Drude-Institut für Festkörperelektronik, 10117 Berlin, Germany.

${ }^{\ddagger}$ Corresponding author: leonhard.grill@uni-graz.at
}

[26]). Porphyrin derivatives are well-studied molecular compounds that are important in biological processes [27] and solar energy conversion [28]. Their properties upon adsorption on surfaces have been intensely studied over the last years [29]. In previous work, we identified porphyrin molecules with differing apparent STM height (named henceforth as b-TPP and d-TPP due to their "bright" and "dark" STM contrasts). The cause for this was the presence or absence of single gold adatoms beneath individual tetra-phenylporphyrin (TPP) molecules [30]. The gold adatom forms a coordination bond with the porphyrin core, similar to what has been suggested as a reaction intermediate in the formation of metalloporphyrins in solution [31] and on a $\operatorname{Ag}(111)$ surface [32], or of TPP [33] or $\mathrm{Br}_{4}$ TPP [34] molecules (i.e., the same as in the present work) on a $\mathrm{Cu}(111)$ surface.

The appearance of our molecules strongly depends on the applied bias voltage: At $-0.5 \mathrm{~V}$ all molecules appear at a similar height, while at around $-1 \mathrm{~V}$ two dark and one bright molecular appearances can be found [Fig. 1(b)]. The two dark molecules could be identified as two tautomers without a gold adatom, while bright molecules have a single gold adatom underneath, causing the shift in the electronic structure [30]. Hence, gold adatoms underneath the molecules are identified from the bright molecular appearance [Fig. 1(b)].

While at low temperatures of $5 \mathrm{~K}$ d-TPP and b-TPP molecules are stable and no spontaneous change of their appearance is observed [30], the molecules switch reversibly between d-TPP and b-TPP at elevated temperatures. An example is shown in Fig. 1(d) where a single $\mathrm{Br}_{4}$ TPP molecule switches from bright to dark during scanning (images are acquired line by line by scanning from the top of the image to the bottom). Due to the low number of switching events at room temperature-about $10^{-4}$ events per second for each (unperturbed) molecule (see Mielke_movie2 [26])—-the process can conveniently be followed with an image repetition time of about $20 \mathrm{~s}$. We exclude chemical changes of the adsorbed molecules as the origin of these changes. Firstly, metalation is known to be absent on $\mathrm{Au}(111)$ at room temperature [35] and proceeds even on the more reactive $\mathrm{Cu}(111)$ surface at temperatures exceeding $400 \mathrm{~K}$ [33]. Secondly, deprotonation [36] of the porphyrin cavity does not occur spontaneously at room temperature [37] and furthermore is 


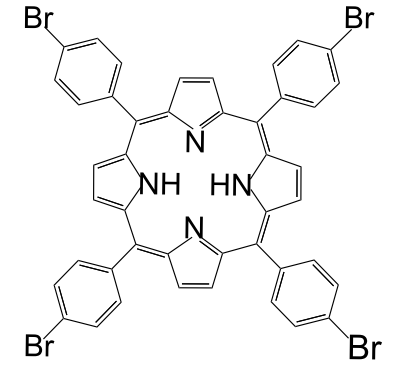

(a)

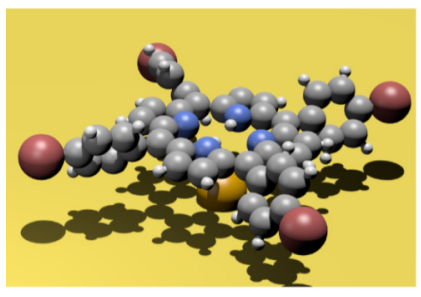

(c)

FIG. 1. (a) Chemical structure and (b) STM image $(12.9 \times$ $\left.12.9 \mathrm{~nm}^{2}, I_{T}=0.14 \mathrm{nA}, \quad U_{\text {tip }}=-0.884 \mathrm{~V}, T=300 \mathrm{~K}\right)$ of tetrabromophenylporphyrin ( $\mathrm{Br}_{4} \mathrm{TPP}$ ) molecules on $\mathrm{Au}(111)$. (c) Scheme of a $\mathrm{Br}_{4}$ TPP molecule on top of a gold adatom. (d) $\mathrm{A} \mathrm{Br}_{4} \mathrm{TPP}$ molecule changing its state while the tip is scanning the area $\left(8 \times 8 \mathrm{~nm}^{2}, I_{T}=0.14 \mathrm{nA}, U_{\text {tip }}=-0.884 \mathrm{~V}, T=300 \mathrm{~K}\right)$.

not in accordance with our observation that the molecules change their appearance when being moved off and onto gold adatoms [30].

By taking many subsequent STM images of the same area, molecules can be studied in different environments (see Mielke_movie1 [26]). When analyzing the switching processes in a highly ordered, defect-free area compared to an area close to a defect, we find a clear enhancement of the switching rate in the vicinity of a defect. Figure 2(a) shows an STM image of an area that includes a domain boundary of the molecular monolayer. In its vicinity, the molecules appear fuzzy due to their rapid switching, but are imaged clearly in the unperturbed monolayer away from the boundary. Note that the herringbone reconstruction is still visible in the STM images, which indicates that the $\mathrm{Au}(111)$ surface is not modified by the molecular layer.

To assess the spatial range of influence of a defect, the number of switching events was counted for individual molecules. The results for molecules with similar distances from a defect were then averaged [Fig. 2(c)], showing a clear transition from a faster to a slower switching rate in the unperturbed monolayer at a large distance of about $10 \mathrm{~nm}$ from the defect, while the total number of bright molecules remains constant. This result indicates that, although the defect itself is highly localized in the STM image, its influence on the switching rate extends even to the fifth molecule at a distance of about $8 \mathrm{~nm}$ (the same behavior was also found for step edges or terrace defects). This is much more than in other molecular processes that depend on the local environment, for instance, molecular switches [20] or molecular cascades [38].
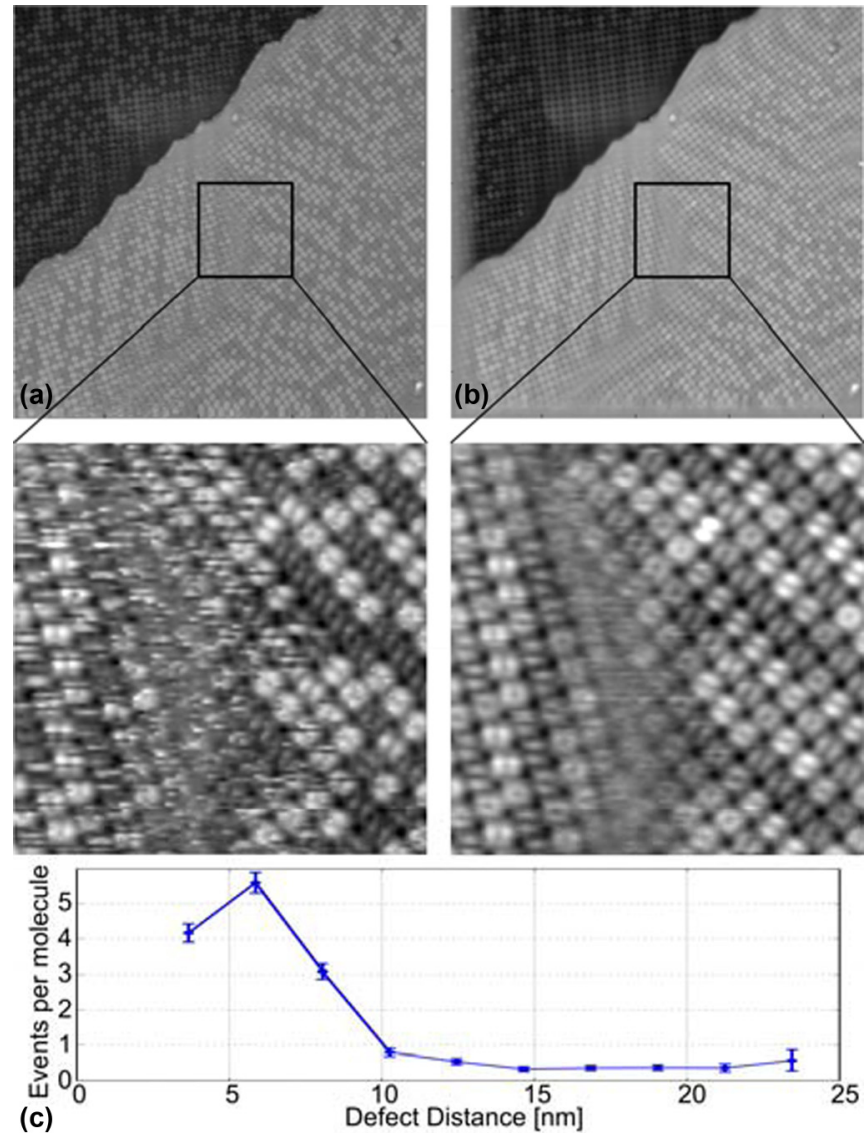

FIG. 2. (a) Single frame from Mielke_movie1 (Supplemental Material [26]) and (b) the average image of the full movie $(87.5 \times$ $87.5 \mathrm{~nm}^{2}, U_{\text {tip }}=-1.25 \mathrm{~V}, I=0.13 \mathrm{nA}, T=300 \mathrm{~K}, 108 \mathrm{~s} /$ frame) . (c) Number of switching events per molecule (for Mielke_movie2 [26]) with $U_{\text {tip }}=-884 \mathrm{mV}, I=0.14 \mathrm{nA}, T=300 \mathrm{~K}, 81 \mathrm{~s} /$ frame. The data point closest to the defect is probably an artifact, caused by the difficulty to correctly assign the molecular state (bright or dark) for rapidly switching molecules at small defect distances. The error bars denote only the $\sqrt{N}$ counting error and not a combined uncertainty.

Importantly, the fuzzy appearance is not caused by rotating molecules, similar to highly mobile porphyrin derivatives on $\mathrm{Cu}(111)$ [33], as the molecules would appear as rings [39], which is not the case. In order to also exclude molecular diffusion as the origin of the streaky molecular appearance, we analyzed the fast switching in the vicinity of defects [as seen in a single frame of the movie, Fig. 2(a)]. However, when all frames of the movie are summed [Fig. 2(b)], the individual molecules in the defect regions become visible again. This confirms that molecular diffusion can be excluded, as in that case the area would appear flat, and the fuzzy appearance is caused by a continuous switching between bright and dark.

In areas unperturbed by defects (Mielke_movie2 [26]) switching is a rather rare event. Figures 3(a) and 3(b) show two subsequent images with two switching molecules. The switching event is visualized more clearly in difference images, where subsequent images are subtracted [Fig. 3(c)]. By analyzing many subsequent images, we found that switching events of opposite direction often occur close to each other, indicating a 

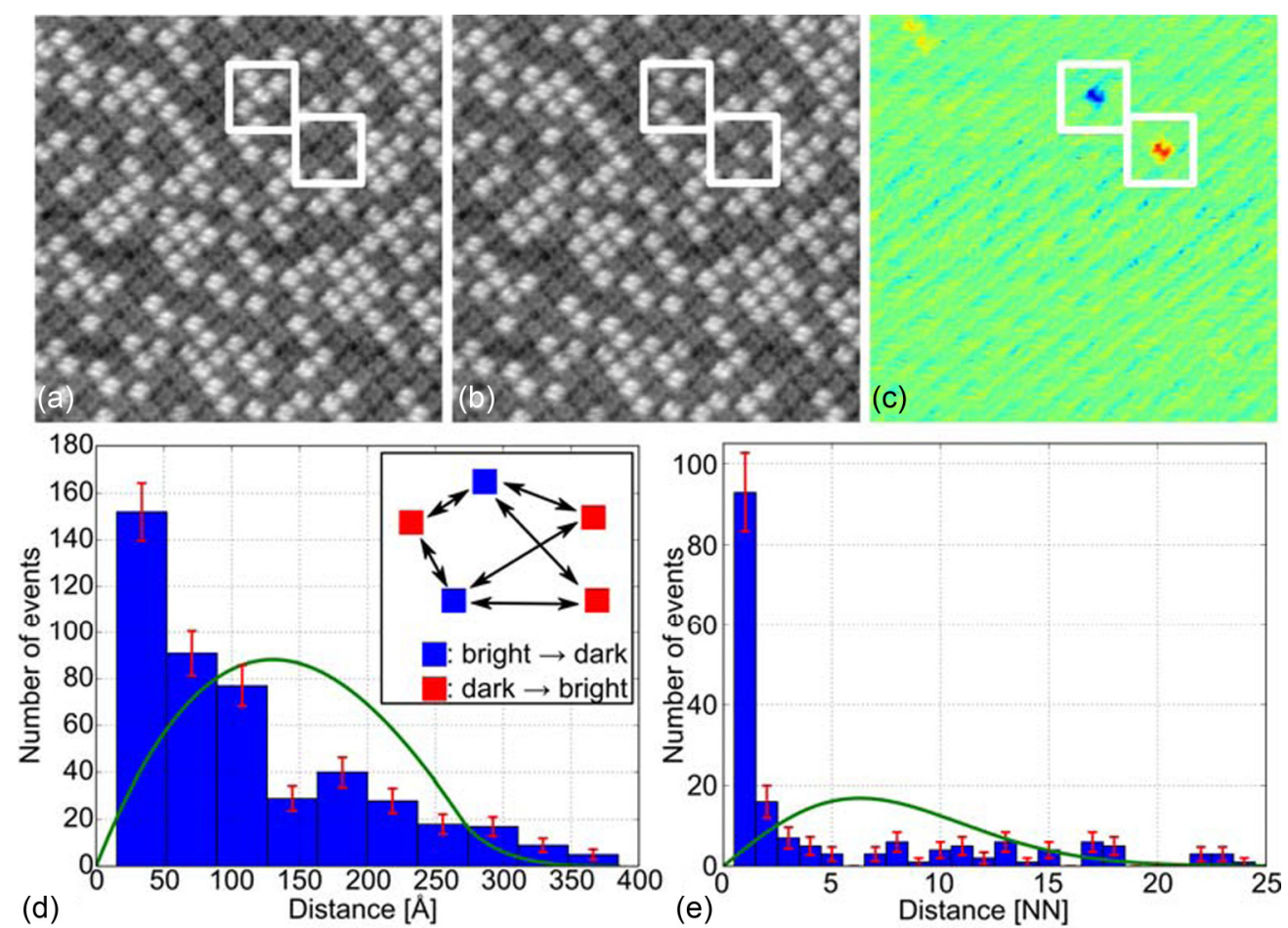

FIG. 3. (a,b) Subsequent STM images (temporal distance $=81 \mathrm{~s})$ of the same area at room temperature $\left(29.8 \times 29.8 \mathrm{~nm}^{2}, U_{\text {tip }}=\right.$ $-0.884 \mathrm{~V}, I_{T}=0.14 \mathrm{nA}, T=300 \mathrm{~K}, 81 \mathrm{~s} /$ frame) and (c) their difference image. Colors as in the inset of (d). (d) Distance distribution of switching events of opposite direction (according to inset), as obtained from difference images (Mielke_movie2_diff [26]). Green line: Distance distribution of uniformly distributed random points in a square [40,50]. (e) Distance distribution of nearest switching events of opposite direction [same movie as in (d)] shown in units of the nearest-neighbor distance. Green line: nearest-event distribution for independent events [51].

spatial correlation between adjacent molecules. Note that this is clearly distinct from previously reported brightness changes where no spatial correlation has been observed, for instance, for $\mathrm{C}_{60}$ on $\mathrm{Ag}$ islands [9].

To quantify this correlation, we determined in every difference image all distances between switching events in opposite direction in defect-free areas. Their histogram (i.e., distances between red and blue spots, but not between spots of the same color) is shown in Fig. 3(d) in comparison with a curve for spatially uncorrelated events (i.e., the distance distribution of random points in a square [40]). Interestingly, there is a pronounced difference between the measured and the random distribution, with a clear preference for switching events of closely spaced molecules, indicating that switching of opposite direction preferentially occurs in close proximity, much closer than expected for random events. Moreover, when analyzing only the distance distribution to the nearest event [Fig. 3(e)] of opposite direction, most of them take place in the direct neighboring molecule. In other words, if one molecule changes from bright to dark, it is very likely that an adjacent molecule (previously dark) becomes bright.

To study the role of direct nearest-neighbor coupling on the switching and to test whether a covalent bond between two porphyrin units influences the switching behavior, we conducted the same experiments with TPP dimers (Fig. 4) that were created on $\mathrm{Au}(111)$ as demonstrated previously [41]. As can be seen in Fig. 4(a) (obtained at $5 \mathrm{~K}$ ), both sides of the dimers are still able to adopt the dark and the bright configuration and can be switched with voltage pulses at low temperatures, where again (as for individual molecules; see Fig. 6) switching from bright to dark is easily achievable, but the opposite direction happens only rarely. At room temperature [Fig. 4(b)], thermal switching can be followed by repeated imaging of the same surface area.

Qualitatively, the switching of the dimers is the same as for the monomers: The distribution of dark and bright within the dimers is completely random [Fig. 4(c)], indicating that there is no communication between the two sides of the dimer and the distance distribution of switching events of opposite direction [similarly as for $\mathrm{Br}_{4}$ TPP in Fig. 3(d)] also shows a clear preference for close switching events as compared to the random distribution [Fig. 4(d)]. Furthermore, the switching speed is also increased in the vicinity of defects. After having understood that the bright state of the monomers is created by the presence of a Au adatom underneath the molecule, the similarity of the dimer behavior is no surprise and confirms that the bright state in the dimers also has to be created by an underlying adatom. The covalent linking between two molecules within a dimer seems to play no role for their behavior and no enhanced correlation between the covalently coupled molecules was observed. Thus, chemical bonds do not influence the switching behavior of the involved molecules. The correlation of opposite switching events in monomer islands (Fig. 3) is most likely caused by exchanging native adatoms, which are the origin of the bright appearance and are additionally known to diffuse at room temperature over 

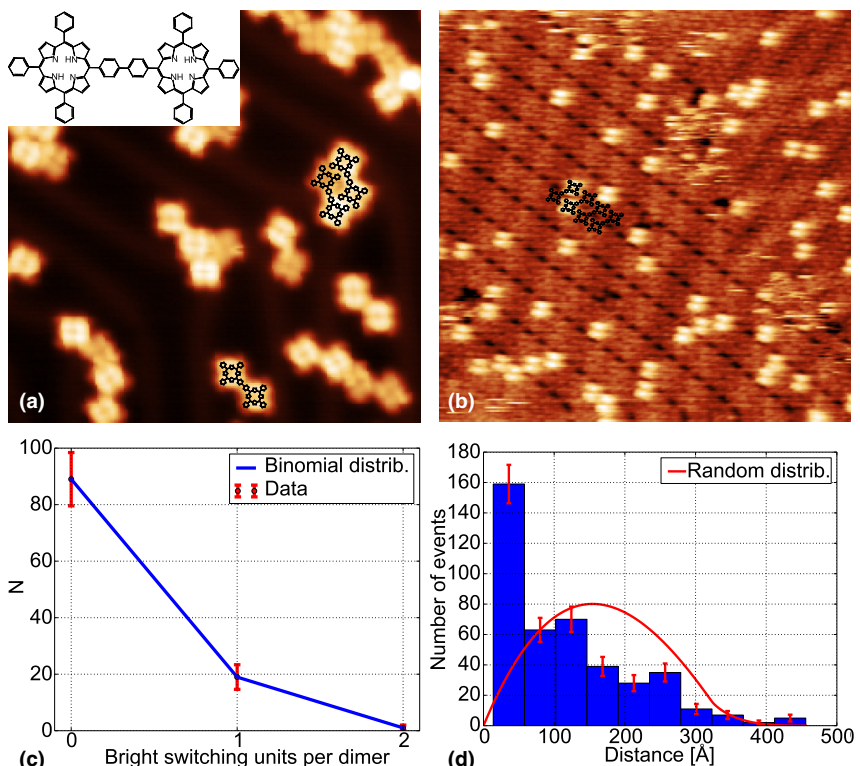

FIG. 4. TPP dimers on $\mathrm{Au}(111)$, created by on-surface synthesis [41]. (a) Appearance of a submonolayer coverage at $5 \mathrm{~K}$ with the dimer's chemical structure in the inset. (b) Full monolayer at room temperature, mixed with BrTPP monomers but segregated into separate domains. (c) Number of bright TPP units per dimer, compared to the corresponding binomial distribution. (d) Distance distribution of switching events of opposite direction as shown in Fig. 3(e) for $\mathrm{Br}_{4} \mathrm{TPP}$ monomers. Image parameters: (a) $22.0 \times 22.0 \mathrm{~nm}^{2}, U_{\text {tip }}=-1.3 \mathrm{~V}, I=0.61 \mathrm{nA}, T=5 \mathrm{~K}$, (b) $37.5 \times$ $37.5 \mathrm{~nm}^{2}, U_{\text {tip }}=-0.884 \mathrm{~V}, I=0.03 \mathrm{nA}, T=300 \mathrm{~K}, 33 \mathrm{~s} /$ frame.

the clean $\mathrm{Au}(111)$ surface [42]. Since bright molecules mark the location of gold adatoms beneath the molecular overlayer, switching likely reflects the motion of a single adatom from one molecule to the next. Moreover, the observation that the switching is qualitatively the same for dimers excludes $\mathrm{Br}$ atoms as the origin of the observed phenomenon. This is because, after sample annealing to $520 \mathrm{~K}$ to produce dimers, $\mathrm{Br}$ atoms are known to be absent [43].

The correlation between switching events of unperturbed molecules (Fig. 3) is in very good agreement with this interpretation. In such cases, an adatom thermally diffuses away from a specific molecule, which consequently switches from bright to dark, and finds a stable adsorption site underneath a neighboring molecule that accordingly turns from dark to bright. This is evident when counting the molecules that switch in either direction between two subsequent frames [Fig. 5(a)]. In most cases the number of dark $\rightarrow$ bright (and hence upswitching) molecules correlates strongly with the number of oppositely down-switching molecules, due to adatom hopping from one molecule to another. If this was true for all switching events, the difference would be constantly zero. However, it scatters around zero, which probably means that in such cases the adatoms remain adsorbed in between the molecules, being invisible for STM imaging (at least until they continue the diffusion), or enter or leave the imaged area.

Although the precise mechanism cannot be determined from our STM observations, we presume that, for instance, a slight lifting of a molecular side group or a weak lateral distortion of Br-phenyl side groups occurs during the adatom
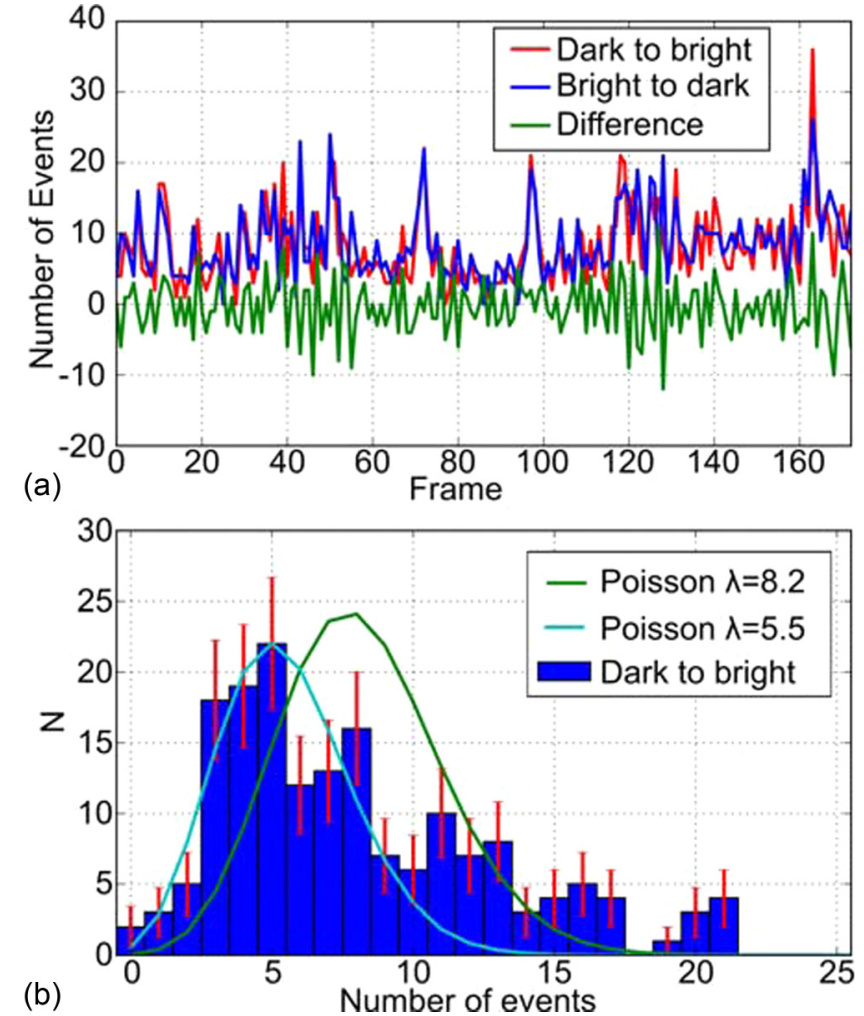

FIG. 5. (a) Number of up- (red) and down- (blue) switching molecules over time within one movie (Mielke_movie3 [26]) $\left(U_{\text {tip }}=\right.$ $-0.302 \mathrm{~V}, I_{T}=0.11 \mathrm{nA}, T=300 \mathrm{~K}, 42 \mathrm{~s} /$ frame) and the difference between both values (green line). (b) Histogram for the number of events between two subsequent frames for $\mathrm{d}-\mathrm{Br}_{4} \mathrm{TPP} \rightarrow \mathrm{b}-\mathrm{Br}_{4} \mathrm{TPP}$. For comparison the Poisson distribution for the observed average number of events between two frames $(\lambda=8.2)$ and the Poisson distribution for $\lambda=5.5$ events, scaled to match the peak height, are shown.

motion from one molecule to another. Accordingly, less ordered molecular assemblies could result in an enhanced molecular flexibility that consequently favors such adatom hopping processes. Our results in Fig. 2 seem to suggest that such a disorder, i.e., slight deviation of the molecular orientation and/or position from the ideal arrangement, extends much further than the size of the defect itself.

Additionally, we observe that in some cases a large number of molecules suddenly switch together (e.g., after frame 35 or 36 in Mielke_movie2_diff; see Supplemental Material [26]). These collective events, evident as high peaks in Fig. 5(a), are typically correlated as they appear simultaneously for both switching directions. Hence, when many molecules become dark (adatoms move away), also many molecules become bright (adatoms move underneath them). Note that this is a simultaneous motion and not a generation of new adatoms. These collective events are even more evident in the histogram for the number of events [Fig. 5(b)]. If the hopping events of the adatoms - and hence both connected switching events-were independent from each other, the number of events for each would follow a Poisson distribution. However, the experimental data do not agree at all with Poisson statistics [green line in Fig. 5(b)] when using the observed 

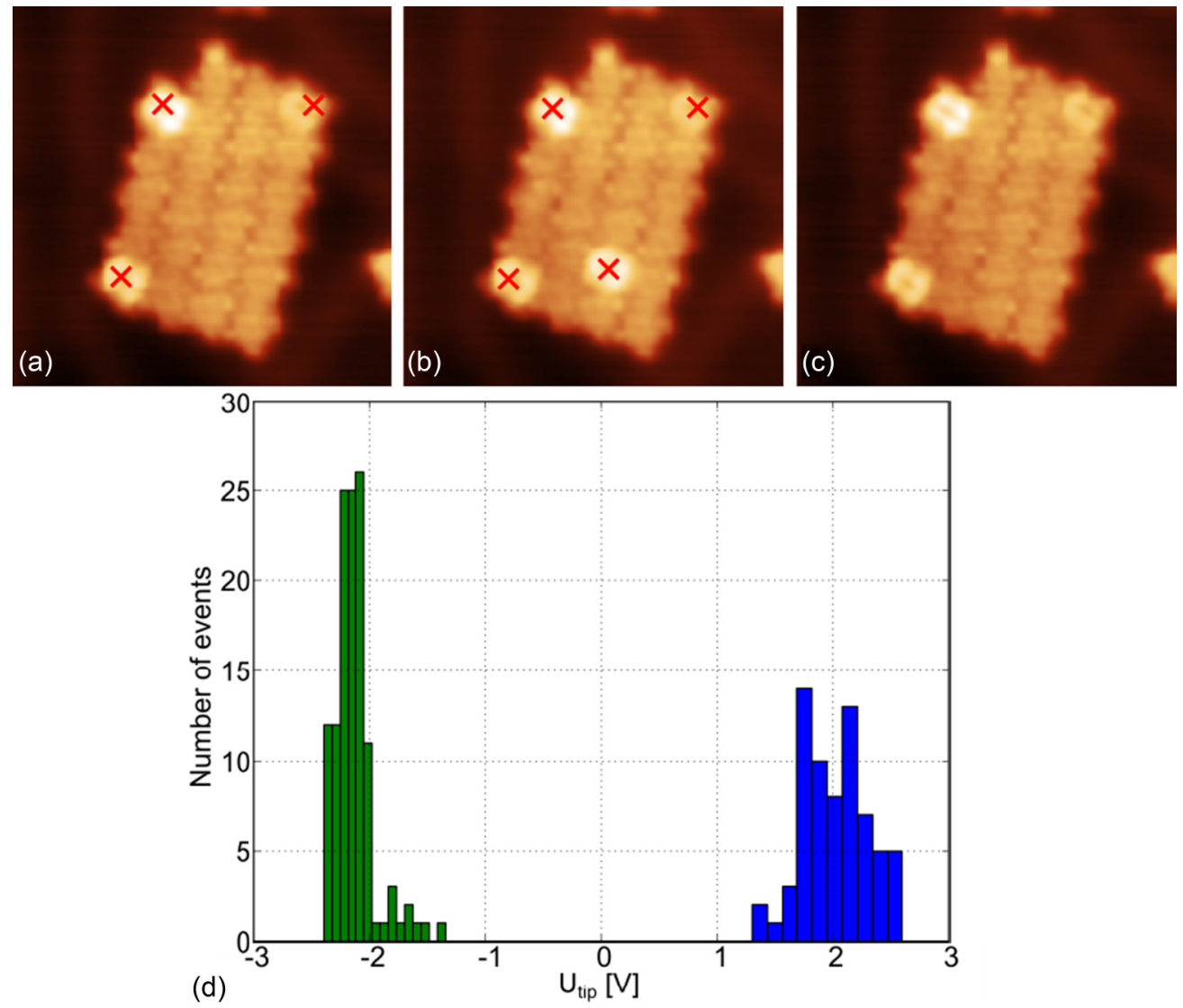

FIG. 6. Switching the appearance of individual molecules by voltage pulses. (a-c) STM images of reversible, tip-induced switching of TPP molecules (between dark and bright) on $\mathrm{Au}(111)$ at $6 \mathrm{~K}$. Voltage pulses were applied in the positions marked with a red cross, 0 to $-1.7 \mathrm{~V}$ in (a), 0 to $-1.8 \mathrm{~V}$ in (b). Image parameters: $12.5 \times 12.5 \mathrm{~nm}^{2}, U_{\text {tip }}=-1 \mathrm{~V}, I=0.31 \mathrm{nA}, T=5.6 \mathrm{~K}$. (d) Histogram ( $\left.n=190\right)$ of successful switching events (from bright to dark) as a function of the bias voltage, which was determined by ramping up the bias voltage from $0 \mathrm{~V}$ up to the bias voltage at which the molecule changes its state. As at these voltages of about $\pm 2 \mathrm{~V}$, dark and bright molecules cannot be distinguished, the state of the molecule needs to be checked in between by shortly returning to a bias of $-1 \mathrm{~V}$.

average number of events as input $(\lambda=8.2$ [26]). If the Poisson distribution is, however, adjusted to the maximum of the measured distribution [by using $\lambda=5.5$; cyan line in Fig. 5(b)], there are a substantial number of data points for large numbers of events $(>10)$ beyond the Poisson statistics. This indicates two kinds of switching processes: first, thermal random switching with low activity (following a Poisson distribution) and second, in addition, the collective switching of many molecules between two frames. These collective "burst" events, leading to a deviation from the Poisson distribution for more than about ten events/frame, indicate a deviation from the assumption of independently diffusing adatoms. Importantly, it appears always in a confined area of the surface (see Mielke_movie2_diff in Supplemental Material [26]). We suggest that the presence of other adsorbates triggers the adatom diffusion, as observed for enhanced atomic or molecular diffusion [44-47], and more specifically for $\mathrm{FeO}$ where the presence of water molecules stimulates the cascadelike motion of hydrogen atoms [48]. We have tested whether water molecules from the rest gas in the vacuum chamber play such a role here [26], but could not find any evidence. Although the origin of this effect is currently unknown, we believe that the experimental observations are clear and that other residual gas molecules or even molecular fragments, which might be sublimed from the evaporator in very small amounts, could also be responsible.

Similar to the thermally induced process of $\mathrm{Br}_{4} \mathrm{TPP}$ molecules, switching between b-TPP and d-TPP molecules can also be induced by voltage pulses from the STM tip at cryogenic temperatures of about $6 \mathrm{~K}$ (Fig. 6). Due to the rare occurrence, no statistics for switching from dark to bright could be obtained and only switching from bright to dark could be used to determine the threshold voltages [Fig. 6(d)]. A reversible process is, for instance, shown by the molecule in the lower center of the island in Figs. 6(a)-6(c) that is switched from dark to bright and back to dark. Tip-induced switching occurs at both bias polarities at threshold voltages of about $\pm 2 \mathrm{~V}$ [Fig. 6(d)]. As neither the highest occupied molecular orbital (HOMO) nor the lowest unoccupied molecular orbital (LUMO) of the bright molecular state is found at these voltages [30], resonant tunneling into the orbitals can be excluded as the cause for the switching.

The yields of the two processes are, however, not equivalent and b-TPP $\rightarrow$ d-TPP switching (yield of about $88 \%$ at a bias voltage of $\pm 2.3 \mathrm{~V}$ ) is much more efficient than in the reverse direction (with a yield of less than $1 \%$ at the same bias voltage and similarly low yields when increasing the bias voltage up to the onset of molecular dissociation). The different switching 
yields are in very good agreement with our interpretation of $\mathrm{Au}$ adatoms as the origin of the b-TPP molecules, because the STM voltage pulses cause adatom hopping underneath the molecules, similar to the manipulation of single atoms [49]. Accordingly, it is rather simple to induce hopping of a $\mathrm{Au}$ adatom away from a b-TPP molecule underneath the tip, but it is of course much more difficult (and consequently a rare event) to cause adatom hopping towards a d-TPP molecule underneath the tip as an adatom must be present in its close vicinity.

In conclusion, we present an in-depth analysis of metal atom diffusion at a molecule-metal interface and provide solid experimental evidence for the emergence of "activity bursts", the sudden appearance of collective switching events in certain localized regions. Such synergistic effects should influence key properties such as catalytic activity and selectivity as well as charge transport, suggesting adatoms as an attractive option to tailor and control molecule-metal interfaces. While the diffusion is on the one hand induced thermally, it can also be caused by controlled voltage pulses from the STM tip where the different yields for the two switching processes (between dark and bright appearance) are in agreement with the process of adatom motion. Future work will be devoted towards unravelling the structural origin of the observed effect and to exploit it to tune organic-inorganic hybrid systems.

We thank Karsten Horn (Fritz-Haber-Institute Berlin) for the kind experimental support and many useful suggestions during the measurements, and Mats Persson and Felix Hanke (University of Liverpool) for helpful discussions. The European Union (Projects No. 243421 ARTIST and No. 270028 AtMol) is gratefully acknowledged for financial support.
[1] G. Ertl, H. Knözinger, F. Schüth, and J. Weitkamp, Handbook of Heterogeneous Catalysis (Wiley-VCH, Weinheim, 2008).

[2] G. A. Somorjai, K. M. Bratlie, M. O. Montano, and J. Y. Park, J. Phys. Chem. B 110, 20014 (2006).

[3] J. R. Heath and M. A. Ratner, Phys. Today 56, 43 (2003).

[4] M. Vladimirova, M. Stengel, A. D. Vita, A. Baldereschi, M. Böhringer, K. Morgenstern, R. Berndt, and W.-D. Schneider, Europhys. Lett. 56, 254 (2001).

[5] C. Dri, M. V. Peters, J. Schwarz, S. Hecht, and L. Grill, Nat. Nanotechnol. 3, 649 (2008).

[6] K. Pussi, H. I. Li, H. Shin, L. N. Serkovic Loli, A. K. Shukla, J. Ledieu, V. Fournée, L. L. Wang, S. Y. Su, K. E. Marino, M. V. Snyder, and R. D. Diehl, Phys. Rev. B 86, 205406 (2012).

[7] H. Shin, A. Schwarze, R. D. Diehl, K. Pussi, A. Colombier, É. Gaudry, J. Ledieu, G. M. McGuirk, L. N. Serkovic Loli, V. Fournée, L. L. Wang, G. Schull, and R. Berndt, Phys. Rev. B 89, 245428 (2014).

[8] J. A. Gardener, G. A. D. Briggs, and M. R. Castell, Phys. Rev. B 80, 235434 (2009).

[9] H. I. Li, G. J. P. Abreu, A. K. Shukla, V. Fournée, J. Ledieu, L. N. Serkovic Loli, S. E. Rauterkus, M. V. Snyder, S. Y. Su, K. E. Marino, and R. D. Diehl, Phys. Rev. B 89, 085428 (2014).

[10] M. Giesen, Prog. Surf. Sci. 68, 1 (2001).

[11] R. Yamachika, M. Grobis, A. Wachowiak, and M. F. Crommie, Science 304, 281 (2004).

[12] L. Gross, K.-H. Rieder, F. Moresco, S. M. Stojkovic, A. Gourdon, and C. Joachim, Nat. Mater. 4, 892 (2005).

[13] R. Raval, S. Haq, F. Hanke, M. S. Dyer, M. Persson, P. Iavicoli, and D. Amabilino, J. Am. Chem. Soc. 133, 12031 (2011).

[14] F. Hanke, S. Haq, R. Raval, and M. Persson, ACS Nano 5, 9093 (2011).

[15] L. Dong, Q. Sun, C. Zhang, Z. Li, K. Sheng, H. Kong, Q. Tan, Y. Pan, A. Hu, and W. Xu, Chem. Commun. 49, 1735 (2013).

[16] F. Rosei, M. Schunack, P. Jiang, A. Gourdon, E. Lagsgaard, I. Stensgaard, C. Joachim, and F. Besenbacher, Science 296, 328 (2002).

[17] L. Grill, K.-H. Rieder, F. Moresco, S. Stojkovic, A. Gourdon, and C. Joachim, Nano Lett. 5, 859 (2005).
[18] L. Grill, F. Moresco, P. Jiang, C. Joachim, A. Gourdon, and K.-H. Rieder, Phys. Rev. B 69, 035416 (2004).

[19] W.-H. Soe, C. Manzano, N. Renaud, P. de Mendoza, A. D. Sarkar, F. Ample, M. Hliwa, A. M. Echavarren, N. Chandrasekhar, and C. Joachim, ACS Nano 5, 1436 (2011).

[20] T. Kumagai, F. Hanke, S. Gawinkowski, J. Sharp, K. Kotsis, J. Waluk, M. Persson, and L. Grill, Nat. Chem. 6, 41 (2014).

[21] T. Zambelli, J. Wintterlin, J. Trost, and G. Ertl, Science 273, 1688 (1996).

[22] A. Saywell, J. Schwarz, S. Hecht, and L. Grill, Angew. Chem. Int. Ed. 51, 5096 (2012).

[23] M. Schunack, T. R. Linderoth, F. Rosei, E. Laegsgaard, I. Stensgaard, and F. Besenbacher, Phys. Rev. Lett. 88, 156102 (2002).

[24] F. Rosei, M. Schunack, Y. Naitoh, P. Jiang, A. Gourdon, E. Laegsgaard, I. Stensgaard, C. Joachim, and F. Besenbacher, Prog. Surf. Sci. 71, 95 (2003).

[25] R. Otero, F. Hümmelink, F. Sato, S. B. Legoas, P. Thostrup, E. Laegsgaard, I. Stensgaard, D. S. Galvao, and F. Besenbacher, Nat. Mater. 3, 779 (2004).

[26] See Supplemental Material at http://link.aps.org/supplemental/ 10.1103/PhysRevB.94.035416 for supplemental movie files and experimental details on the methods, the time dependence and spatial distribution of the switching process, the collective events, and the role of tip effects, as well as a comparison of $\mathrm{TPP}$ and $\mathrm{Br}_{4}$ TPP molecules.

[27] L. R. Milgrom, The Colours of Life. An Introduction to the Chemistry of Porphyrins and Related Compounds (Oxford University Press, Oxford, 1997).

[28] L.-L. Li and E. W.-G. Diau, Chem. Soc. Rev. 42, 291 (2012).

[29] J. M. Gottfried, Surf. Sci. Rep. 70, 259 (2015).

[30] J. Mielke, F. Hanke, M. V. Peters, S. Hecht, M. Persson, and L. Grill, J. Am. Chem. Soc. 137, 1844 (2015).

[31] E. B. Fleischer and J. H. Wang, J. Am. Chem. Soc. 82, 3498 (1960).

[32] F. Buchner, K. Flechtner, Y. Bai, E. Zillner, I. Kellner, H.-P. Steinrück, H. Marbach, and J. M. Gottfried, J. Phys. Chem. C 112, 15458 (2008).

[33] S. Ditze, M. Stark, M. Drost, F. Buchner, H.-P. Steinrück, and H. Marbach, Angew. Chem. Int. Ed. 51, 10898 (2012). 
[34] C. M. Doyle, S. A. Krasnikov, N. N. Sergeeva, A. B. Preobrajenski, N. A. Vinogradov, Y. N. Sergeeva, M. O. Senge, and A. A. Cafolla, Chem. Commun. 47, 12134 (2011).

[35] Y. Li, J. Xiao, T. E. Shubina, M. Chen, Z. Shi, M. Schmid, H.-P. Steinrück, J. M. Gottfried, and N. Lin, J. Am. Chem. Soc. 134, 6401 (2012).

[36] L. Smykalla, P. Shukrynau, C. Mende, T. Rüffer, H. Lang, and M. Hietschold, Surf. Sci. 628, 92 (2014).

[37] W. Auwärter, K. Seufert, F. Bischoff, D. Ecija, S. Vijayaraghavan, S. Joshi, F. Klappenberger, N. Samudrala, and J. V. Barth, Nat. Nanotechnol. 7, 41 (2012).

[38] A. J. Heinrich, C. P. Lutz, J. A. Gupta, and D. M. Eigler, Science 298, 1381 (2002).

[39] J. K. Gimzewski, C. Joachim, R. R. Schlittler, V. Langlais, H. Tang, and I. Johannsen, Science 281, 531 (1998).

[40] A. M. Mathai, P. Moschopoulos, and G. Pederzoli, Rend. Circolo Mat. Palermo 48, 163 (1999).

[41] L. Grill, M. Dyer, L. Lafferentz, M. Persson, M. V. Peters, and S. Hecht, Nat. Nanotechnol. 2, 687 (2007).

[42] C. A. Lang, M. M. Dovek, J. Nogami, and C. F. Quate, Surf. Sci. 224, L947 (1989).
[43] S. A. Krasnikov, C. M. Doyle, N. N. Sergeeva, A. B. Preobrajenski, N. A. Vinogradov, Y. N. Sergeeva, A. A. Zakharov, M. O. Senge, and A. A. Cafolla, Nano Res. 4, 376 (2011).

[44] B. G. Briner, M. Doering, H.-P. Rust, and A. M. Bradshaw, Science 278, 257 (1997).

[45] G. L. Kellogg, Phys. Rev. B 55, 7206 (1997).

[46] S. Horch, H. T. Lorensen, S. Helveg, E. Laegsgaard, I. Stensgaard, K. W. Jacobsen, J. K. Norskov, and F. Besenbacher, Nature 398, 134 (1999).

[47] K. L. Wong, B. V. Rao, G. Pawin, E. Ulin-Avila, and L. Bartels, J. Chem. Phys. 123, 201102 (2005).

[48] L. R. Merte, G. W. Peng, R. Bechstein, F. Rieboldt, C. A. Farberow, L. C. Grabow, W. Kudernatsch, S. Wendt, E. Laegsgaard, M. Mavrikakis, and F. Besenbacher, Science 336, 889 (2012).

[49] J. A. Stroscio, F. Tavazza, J. N. Crain, R. J. Celotta, and A. M. Chaka, Science 313948 (2006).

[50] J. Mielke, Ph.D. thesis, Freie Universität Berlin, 2013.

[51] H. C. Tuckwell, Elementary Applications of Probability Theory, 2nd ed. (Chapman and Hall/CRC, London, 1995). 